\title{
Note
}

\section{A novel partial male sterility resource in bread wheat}

\author{
Maxime Trottet, Denise Deffains and Joseph Jahier*
}

INRA, UMR APBV, Plant Genetics \& Biotechnology, BP 35327, 35653 Le Rheu Cedex, France

\begin{abstract}
A plant of the wheat cultivar Moulin grown in a greenhouse was found to be completely sterile. The study of the $F_{1}$ and $F_{2}$ offspring of the plant pollinated by Moulin showed that in field conditions the sterility was partial (fertility reduced by $91.6 \%$ with only a few completely sterile plants), recessive and monogenic. The $\mathrm{F}_{1}$ crosses of the mutant Moulin with $M s 1 \mathrm{~b} / \mathrm{ms} 1 \mathrm{~b}$ Sirocco and Probus monosomic 4B showed that the gene for sterility is not located in chromosome $4 \mathrm{~B}$ and hence cannot be allelic to the recessive allele $m s 1 b$ which confers complete male sterility in wheat. The expression of sterility was demonstrated in wheat backgrounds with high levels of fertility. This is the first time partial-monogenic sterility is reported in wheat. This kind of sterility is of no interest for producing wheat $F_{1}$ hybrids. However, it might be of interest for recurrent selection, or dynamic management of genetic resources where limited intermating is required, as well as in the study of genes involved in microsporogenesis.
\end{abstract}

Key Words: male sterility, wheat, mutation, chromosomal location.

\section{Introduction}

Eleven mutations conferring complete male sterility have been located on the wheat genome (see http://www.shigen. nig.ac.jp/wheat/komugi/genes/symbolClassList.jsp). Three of them are dominant: Ms2 (Liu and Deng 1986), $M s 3$ (Maan et al. 1987) and Ms4 (Maan and Kianian 2001). Among the eight recessive mutations, seven are allelic on chromosome 4B ( $m s 1 a$ to $m s l g$ ) and the eighth, $m s 5$, is located on chromosome 3A (Klindworth et al. 2002). Other sources of male sterility have been found but their genetic determinism and chromosomal locations were not achieved, e.g. Bingham (1967) and Krupnov (1968).

Reports of partial fertility are rare. Jan and Qualset (1977), Gill and Anand (1970) and Athwal et al. (1967) described three sources of partial sterility conditioned by three recessive genes; the plants with the three ms genes had a seed set of about 5\%. Gill and Anand (1970) suggested that a parent with $5 \%$ seed set could be used as the female for hybrid seed production.

The cultivar Moulin (Maris Widgeon//Yecora/Ciano 67/ 3/Hobbit) was bred by The Plant Breeding Institute (UK) and registered in the French Catalogue in 1984. A plant of this cultivar grown in a greenhouse was found completely sterile. The objectives of the present study were to: $i$ ) assess the level of male sterility (or self-fertility) in normal crop

Communicated by T. Nishio

Received August 18, 2010. Accepted October 31, 2010.

*Corresponding author (e-mail: joseph.jahier@rennes.inra.fr) conditions in the field, ii) determine its inheritance, iii) because, at the beginning of the study, the three known recessive genes for male sterility were located on chromosome $4 \mathrm{~B}$, crosses were made to validate its putative localization on $4 \mathrm{~B}$, and in that case, possible identity with $m s 1 b$, and $i v$ ) determine expression of the mutation in different backgrounds.

\section{Materials and Methods}

Hereafter, cv-Moulin designates the cultivar Moulin, msMoulin the sterile mutant of cv-Moulin, msMs-Moulin the plant heterozygous for the sterility gene.

The initial ms-Moulin plant, whose meiotic behaviour at the MI stage of meiosis was analysed using a standard technique, was crossed with cv-Moulin. In the $\mathrm{F}_{2}$ generation of the cross, the partially sterile plants were bagged. The $F_{3}$ generation was grown to check for homozygosity of partial male sterility. The sterility of ms-Moulin was assessed in the $\mathrm{F}_{4}$ generation in the field. The number of grains on the whole ear and the two lateral flowers and the number of spikelets per ear were evaluated at maturity after harvest. All seeds from sterile plants used in this study came from bagged ears. We also assessed pollen viability using acetocarmine staining as described by Delourme (1996).

Sirocco is a near-isogenic line of cv. Hobbit with the recessive $m s 1 b$ gene from a mutant of $\mathrm{cv}$. Probus. It is a mixture of plants which are heterozygous and homozygous for $m s 1 b$ (created by J. Bingham, PBI, Cambridge, UK). Heterozygous plants were crossed as male to ms-Moulin. 
Seeds of Probus monosomic for chromosome 4B (Probus 4B) were kindly provided by Dr. Kleijer (Switzerland). Chromosome counting enabled screening of monosomic plants which were then crossed to heterozygous plants of both msMs-Moulin and fertile $(M s 1 b / m s 1 b)$ plants of Sirocco.

ms-Moulin and cv-Moulin were crossed to the five cultivars PR22R28, Orvantis, Isengrain, Apache, Caphorn. These cultivars, which have been widely grown in France in the last decade, were bred by different breeding companies (Pioneer, Benoist, Desprez, Nickerson, PBI/Monsanto respectively). They are assumed to be weakly related. $F_{1}$ plants of the ten crosses were selfed. The ears of the first tillers of $100 \mathrm{~F}_{2}$ plants per population grown in the field were bagged and the number of spikelets and grains per ear were counted after harvest. The distribution of fertile vs. sterile plants was compared to expected distribution using a chi-square test. Means were compared with Student's t-test.

\section{Results and Discussion}

\section{Likelihood of a mutation conferring sterility}

The sterile plant was initially found among a batch of fully fertile plants of cv-Moulin. In order to check that the sterility was not due to aneuploidy or meiotic disturbances, anthers were collected on one of the last tillers at the MI stage of meiosis. The number of chromosomes was 42 and meiotic pairing was regular, i.e. most of pollen mother cells displayed 21 bivalents. This suggested a mutation conferred sterility to the plant (dominant mutation) or to the mother plant (recessive mutation). The plant was crossed to $\mathrm{cv}$. Moulin without difficulty, indicating that female fertility was not altered.

\section{Level of sterility conferred by the mutation}

Plants of the $\mathrm{F}_{3}$ generation of the cross between the initial ms-Moulin plant and cv-Moulin were highly sterile and some were completely sterile, demonstrating that the mutation did not confer complete sterility, and that complete sterility is infrequent. Two bagged ears of 10 plants of ms-Moulin ( $\mathrm{F}_{4}$ generation) and cv-Moulin grown in the field were used to estimate the seed set of the two genotypes (Table 1). On average, cv-Moulin and ms-Moulin yielded 104 (77 to 120 ) and 8.7 (0 to 16) grains per ear respectively, i.e. a reduction of $91.6 \%$ in fertility due to the mutation. The mean number of grains per spikelet was 4.17 and 0.35 for cv-Moulin and ms-Moulin respectively. The mean number of grains per lateral floret was 0.06 in ms-Moulin. Although it was not assessed in cv-Moulin, it can be hypothesised that the sterility would affect the lateral florets to a greater extent.

\section{Recessivity/Dominance}

The $\mathrm{F}_{1}$ plants from the cross between ms-Moulin and cvMoulin were as fertile as the cv-Moulin. Pollen fertility also was evaluated by the percentage of pollen grains stained by acetocarmine in three plants of cv-Moulin, four of msMs-
Table 1. Measurements of fertility in the mutant ms-Moulin and cv-Moulin

\begin{tabular}{|c|c|c|c|c|c|}
\hline & \multirow{2}{*}{$\begin{array}{l}\text { Grains/lateral } \\
\text { floret in } \\
\text { ms-Moulin }\end{array}$} & \multicolumn{2}{|c|}{$\begin{array}{l}\text { Number of grains } \\
\text { per spikelet }{ }^{a}\end{array}$} & \multicolumn{2}{|c|}{$\begin{array}{c}\text { Number of grains } \\
\text { per ear }^{a}\end{array}$} \\
\hline & & ms-Moulin & cv-Moulin & ms-Moulin & cv-Moulin \\
\hline Plant 1 & 0.06 & 0.61 & 4.45 & 15.5 & 114 \\
\hline Plant 2 & 0.02 & 0.11 & 4.33 & 2.5 & 104 \\
\hline Plant 3 & 0.08 & 0.59 & 4.78 & 16.0 & 120 \\
\hline Plant 4 & 0.00 & 0.00 & 3.00 & 0.0 & 77 \\
\hline Plant 5 & 0.00 & 0.28 & 4.21 & 7.0 & 101 \\
\hline Plant 6 & 0.06 & 0.29 & 4.49 & 7.0 & 115 \\
\hline Plant 7 & 0.11 & 0.65 & 4.33 & 16.0 & 104 \\
\hline Plant 8 & 0.14 & 0.52 & 4.67 & 11.0 & 115 \\
\hline Plant 9 & 0.01 & 0.02 & 3.31 & 0.5 & 86 \\
\hline Plant 10 & 0.10 & 0.47 & 4.08 & 12.0 & 102 \\
\hline means & 0.06 & $\mathrm{~m} 1=0.35$ & $\mathrm{~m} 2=4.14$ & $\mathrm{~m} 1=8.75$ & $\mathrm{~m} 2=104$ \\
\hline \multicolumn{2}{|c|}{$p(\mathrm{~m} 1=\mathrm{m} 2)^{b}$} & \multicolumn{2}{|c|}{$<10^{-10}$} & \multicolumn{2}{|c|}{$<10^{-10}$} \\
\hline
\end{tabular}

Moulin and six of ms-Moulin; and the results showed that $78 \%, 71 \%$ and $49 \%$ of pollen grains were fertile in the three populations.

The $\mathrm{F}_{2}$ generation of $\mathrm{ms}$ Moulin $\times$ Moulin was analyzed in the greenhouse and in the field. A first group of plants designated as sterile yielded less than 20 grains per ear in both locations while the others designated as fertile had a more or less normal fertility with at least 60 grains per ear. In the greenhouse, the $F_{2}$ segregated 7 sterile vs. 22 fertile plants, and in the field, 31 sterile vs. 112 fertile plants were observed. Comparisons with a $3: 1$ theoretical distribution unambiguously showed that sterility in ms-Moulin was monogenic and recessive $\left(\chi^{2}=0.0115, p=0.91\right.$ and $\chi^{2}=0.8415$, $p=0.36$ respectively).

\section{Is the mutation allelic to $m s 1 b$ ?}

We hypothesised that the mutation leading to sterility would affect the same gene for recessive sterility ( $m s l b$ on chromosome 4B) as in Sirocco. Negative environmental conditions did not appear to alter the fertility of the plants from the Probus $4 \mathrm{~B} \times \mathrm{msMs}-M o u l i n$, Probus $4 \mathrm{~B} \times M s 1 b$ / $m s 1 b$ Sirocco, and msMs-Moulin $\times M s 1 b / m s 1 b$ Sirocco crosses, according to the observed level of fertility of cvMoulin and Sirocco (Table 2). Sirocco segregated in the expected $1: 1$ (fertile : sterile) ratio.

Chromosomes were counted in 57 plants of the $F_{1}$ between Probus monosomic 4B and heterozygous Sirocco. The 10 plants with 42 chromosomes were all fertile. Among the 47 monosomic plants, 17 were completely sterile. The other 30 plants were fertile with the number of grains per ear ranging from 49 to 75 . This showed that the monosomic plants used in the crosses with msMs-Moulin were undoubtedly monosomic for the $4 \mathrm{~B}$ chromosome, and confirmed that the mutation in Sirocco was located on chromosome 4B.

In the ms-Moulin $\times M s 1 b / m s 1 b$ Sirocco cross, only one 
Table 2. Grain fertility in parental lines and in crosses testing the allelism of the mutation in ms-Moulin with the $m s 1$ gene

\begin{tabular}{|c|c|c|c|c|c|}
\hline & Chromosome countings & Sterility vs. fertility & Number of plants & $\begin{array}{c}\text { Mean number of grains/ } \\
\text { spikelet }\end{array}$ & range \\
\hline cv-Moulin & & & 10 & 4.17 & $3.00-4.78$ \\
\hline \multirow[t]{2}{*}{ Sirocco } & & $\mathrm{F}$ & 9 & 3.49 & $2.48-4.35$ \\
\hline & & $\mathrm{S}$ & 11 & 0.00 & - \\
\hline \multirow{3}{*}{$\begin{array}{l}\text { Probus } 4 \mathrm{~B} \times M s 1 b / m s 1 b \\
\text { Sirocco }\end{array}$} & $2 n=42$ & $\mathrm{~F}$ & 10 & 2.91 & $1.94-3.33$ \\
\hline & $2 n=41$ & $\mathrm{~F}$ & 30 & 2.63 & $2.08-3.38$ \\
\hline & & $\mathrm{S}$ & 17 & 0.00 & \\
\hline \multirow[t]{2}{*}{ Probus $4 \mathrm{~B} \times \mathrm{msMs}-$ Moulin } & $2 n=42$ & $\mathrm{~F}$ & 18 & 3.46 & $2.37-4.02$ \\
\hline & $2 \mathrm{n}=41$ & $\mathrm{~F}$ & 65 & 2.79 & $1.93-3.53$ \\
\hline \multirow[t]{2}{*}{ ms-Moulin $\times$ Sirocco } & & $\mathrm{F}$ & 99 & 4.18 & $2.62-5.36$ \\
\hline & & S & 1 & 0.00 & \\
\hline
\end{tabular}

plant out of 100 was found to be sterile. We concluded that the cause of its sterility was not connected with the sterility of ms-Moulin. If the latter were located on chromosome 4B, about $50 \%$ of the plants would be sterile or at least have a level of fertility lower than the least fertile among the 99 fertile plants (2.62 grains per spikelet).

No monosomic or euploid plant with low fertility was found in the Probus $4 \mathrm{~B} \times$ msMs-Moulin cross. But the mean fertility rate of the 65 monosomic plants (2.79 grains per spikelet, range 1.93-3.70) was significantly lower ( $p=0.001)$ than that of the 18 plants with $2 \mathrm{n}=42$ chromosomes (3.46 grains per spikelet, range 2.37-4.02). This reduction in fertility was likely due to the monosomic state.

\section{Expression of sterility in other genetic backgrounds}

We assessed the fertility of the five $F_{2}$ populations of ms-Moulin $\times$ Isengrain, ms-Moulin $\times$ Orvantis, ms-Moulin $\times$ PR22R28, ms-Moulin $\times$ Caphorn, ms-Moulin $\times$ Apache, and the corresponding $\mathrm{F}_{2}$ with $\mathrm{cv}-\mathrm{Moulin}$ as female parent, as well as their parents. We started by scoring fertility on two batches of 20 random ears per population/genotype. Data on each batch were kept separately. As there was no significant difference between the two batches, the analysis was limited to 40 plants.

The first four cultivars parents of the $F_{2}$ populations: Isengrain, Orvantis, PR22R28 and Caphorn yielded between 3.07 and 3.55 grains per spikelet (data not shown). The fifth cultivar, cv. Apache, which is known for ear narrowing at the apex but with a similar number of spikelets, produced 2.50 grains per spikelet (range: 1.72-2.90). No plant of any of the five cultivars displayed sterility since all the plants had at least 1.72 grains per spikelet.

Fig. 1 shows the distribution of the plants within the $F_{2}$ populations according to their mean number of grains per spikelet. For all male-fertile parents, the distribution of the two corresponding $\mathrm{F}_{2}$ populations (ms-Moulin $\times$ cultivar and cv-Moulin $\times$ cultivar) differed, with more plants with low grain fertility in the $\mathrm{F}_{2}$ that had ms-Moulin as the female parent (Fig. 1A to 1E).

In the parents ms-Moulin and cv-Moulin, the distribu- tions were clearly separated. The maximum for ms-Moulin was 0.88 and the minimum for cv-Moulin was 2.17 (Fig. 1F). The plants in the different $\mathrm{F}_{2}$ populations having $\leq 1.50$ grains were consequently considered as sterile. The number of sterile plants was compared to 10 , the theoretical number of sterile plants under the hypothesis that $25 \%$ were homozygous msms and with a sterility level similar to that of ms-Moulin. Using the chi-square test, the hypothesis was valid in the four sterile $F_{2}$ populations that had Isengrain, Orvantis, PR22R28 or Caphorn as the fertile male parent. In the case of cv. Apache, which produced significantly fewer grains per spikelet than the other four cultivars, the hypothesis was rejected.

\section{Concluding remarks}

A monogenic recessive mutation which conferred partial male sterility occurred in cv-Moulin. In contrast to our initial hypothesis, it was not located on chromosome 4B. Since the beginning of the study, another recessive male sterile gene, $m s 5$, was found on 3AL (Klindworth et al. 2002). So the mutation might be located on that chromosome.

Among the few examples of partial sterility reported in wheat, that of cv-Moulin is the only one to be monogenic. It is clear that it is of no interest for production of wheat $F_{1}$ hybrids since the breeding of $F_{1}$ cultivars presently relies on use of gametocides and since the cost of production of malesterile seeds of ms-Moulin would be too high. However it could be of interest in $i$ ) situations of recurrent selection or of dynamic management where limited intermating is required and ii) in studies of genes involved in microsporogenis and pollen development. In recent years, using findings from model organisms, research has been undertaken to understand the mechanisms that govern the cellular process of meiosis and microsporogenesis in hexaploid wheat (Able et al. 2009). ms-Moulin should be useful in that context. Seeds of ms-Moulin are available on request. 

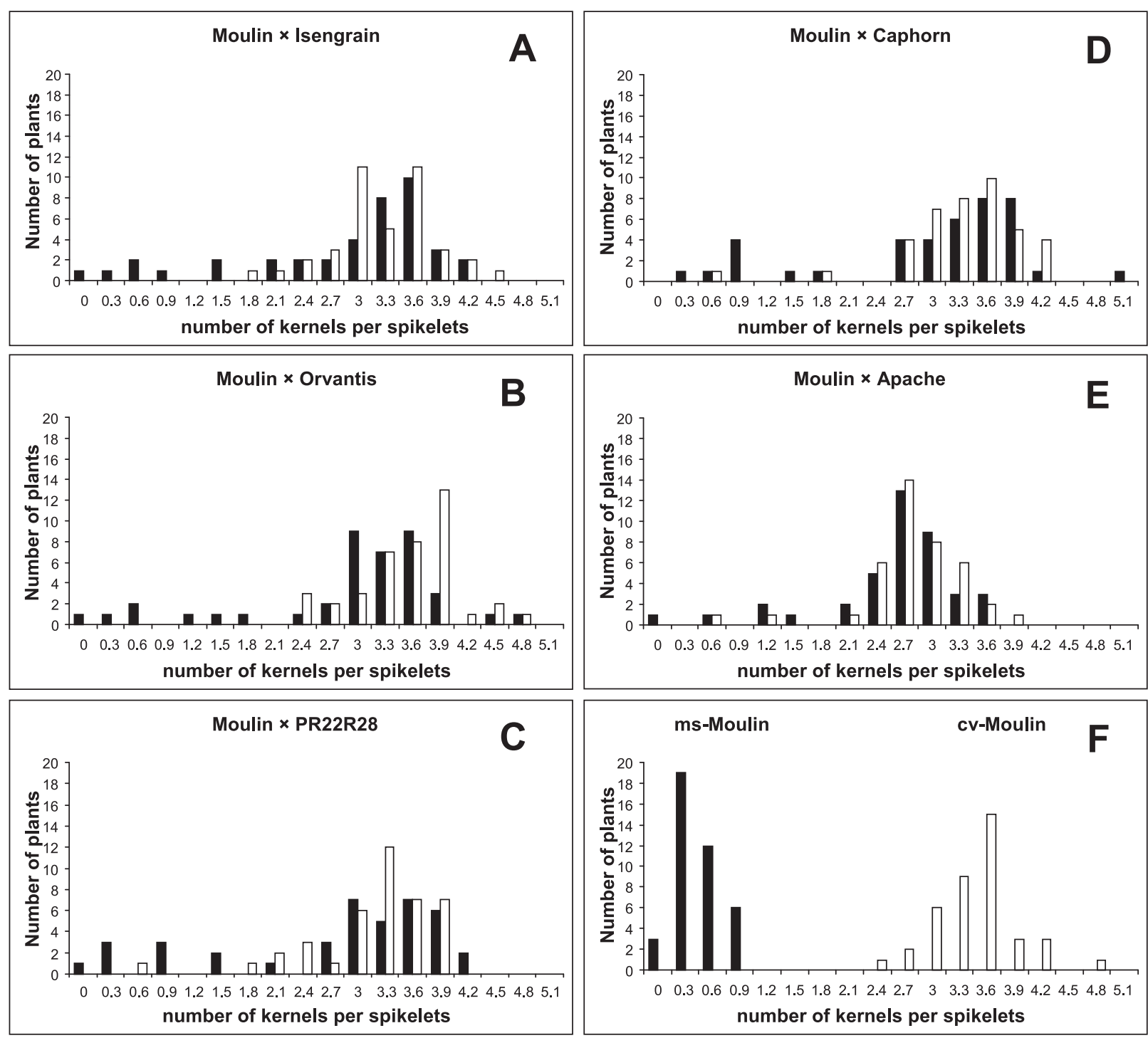

Female parent: ms-Moulin

Female parent: cv-Moulin

Fig. 1. Distribution of fertility of ms-Moulin (male sterile) and cv-Moulin (fertile) (F), and of $F_{2}$ plants from crosses of these two genotypes to Isengrain, Orvantis, PR22R28, Caphorn and Apache (A to E).

\section{Literature Cited}

Able,J.A., W.Crismani and S.A.Boden (2009) Understanding meiosis and the implications for crop improvement. Functional Plant Biology 36: 575-588.

Athwal,D.S., P.S.Phul and J.L.Minocha (1967) Genetic male sterility in wheat. Euphytica 16: 354-360.

Bingham,J. (1967) Report of the Plant Breed. Inst., Cambridge, England. 1966-67: 67.

Delourme, R. (1996) Pollen fertility after staining. In: Jahier,J. (ed.) Techniques of Plant Cytogenetics, Oxford \& IBH Publishing Co. Pvt. Ltd., p. 98.

Gill,B.S. and S.C.Anand (1970) Genetic male sterility for hybrid seed production in wheat. Crop Sci. 10: 385-386.
Jan, C.C. and C.O.Qualset (1977) Genetic male sterility in wheat: inheritance. Crop Sci. 17: 791-794.

Klindworth,D.L., N.D.Williams and S.S.Maan (2002) Chromosomal location of genetic male sterility genes in four mutants of hexaploid wheat. Crop Sci. 42: 1447-1450.

Krupnov,V.A. (1968) Genetic male sterility in wheat (Triticum aestivum L.). Genetika 10: 28-35.

Liu,B.H. and J.Y.Deng (1986) A dominant gene for male sterility in wheat. Plant Breed. 97: 204-209.

Maan,S.S., K.M.Carlson, N.D.Williams and T.Yang (1987) Chromosome arm location and gene centromere distance of a dominant gene for male sterility in wheat. Crop Sci. 27: 494-500.

Maan, S.S. and S.F.Kinian (2001) Third dominant male sterility gene in common wheat. Wheat Info. Serv. 93: 27-31. 